\title{
44560 - AORTO-LEFT ATRIAL FISTULA DETECTED BY POSTOPERATIVE TEE AFTER AORTIC VALVE REPLACEMENT
}

\author{
Farhan Husain, Toronto General Hospital, University Health Network, Toronto, ON, \\ Canada; \\ George Djaiani, Toronto General Hospital, University Health Network; \\ Christopher Feindel, Toronto General Hospital, University Health Network; \\ Jacek Karski, Toronto General Hospital, University Health Network;
}

PURPOSE: Role of Transesophageal Echocardiography (TEE).Aorto-Left Atrial fistula after Aortic Valve Replacement (AVR).

CLINICAL FEATURES: After obtaining patient consent we describe an aorto-left atrial fistula detected by intraoperative TEE following aortic valve replacement for severe aortic stenosis. Communications between the aorta and the chambers of the heart after valve replacement are rare but can be potentially life threatening if not recognized and treated in a timely manner. (1, 2, 3) After obtaining written informed consent a 70-year-old male was scheduled for elective AVR for severe aortic stenosis with an estimated aortic valve area of $0.6 \mathrm{~cm} 2$ with a peak gradient of $64 \mathrm{mmHg}$ and a mean gradient of $35 \mathrm{mmHg}$. Other TEE findings revealed thickening of the mitral valve leaflets with moderate to severe mitral regurgitation (MR) with a functionally grade I/IV left ventricle. Angiography showed a $60-70 \%$ occlusion of the left anterior descending artery disease (LAD). In addition to his cardiac medications he was taking oral medication for Type II Diabetes Mellitus and coumadin for DVT. After induction of general anesthesia the surgeon proceeded to replace the aortic valve with a $25 \mathrm{~mm}$ mechanical prosthesis after extensive debulking of the calcification around the aortic valve area and enlargement of the annulus. The mitral valve was inspected and the anterior mitral valve leaflet repaired with a pericardial patch followed by a bypass graft to the LAD. Prior to separation from bypass a TEE examination was performed to assess the adequacy of valve repair, which revealed a well functioning aortic valve prosthesis with a transvalvular gradient of $20 \mathrm{mmHg}$ and no regurgitation. There was still mild to moderate MR however a new eccentric, posteriorly directed jet was noted. These findings were communicated to the surgeon and a visual inspection revealed a small tear in the calcified area around the aortic annulus, which had been previously debulked, and was communicating with the left atrium. A pericardial patch was deployed to close the defect. A repeat TEE showed that the intervention was successful and the offending jet was not visible anymore. The remainder of the operation was uneventful and the patient was discharged home 6 days after surgery. CONCLUSION: This case report demonstrates a rare surgical complication of aorta-left atrial fistula after AVR, and highlights the importance of intraoperative TEE in identifying pathological findings after valve replacement surgery(4). A routine TEE should be encouraged in patients scheduled for AVR, and a thorough comparison should always be made to the prebypass TEE findings, particularly in patients with significant annular calcification REFERENCES: J Am Soc Echocardiogr 2000, 13:69-72.,J Am Soc Echocardiogr 2002, 15:1409-11. ,CHEST 1997, 111:828-31., J Heart Valve Dis. 2002 Jan; 11(1): 135-8 